\title{
Les enjeux idéologiques des théories évolutionnistes
}

\author{
Alexandre Gefen \\ CNRS - Université Paris 4 Sorbonne
}

En tentant, selon les termes de Jacques Morizot, le "franchissement du gouffre entre matière et conscience" (2012a, p. 89), les sciences cognitives - dans une triade constituée par la psychologie expérimentale, les neurosciences et la théorie de l'information - déploient aujourd'hui une grande variété de niveaux d'analyse et de champs d'expertise, qu'elles s'intéressent aux supports moléculaires, cellulaires, neuronaux, comportementaux, sociaux, ou anthropologiques, de la cognition humaine et donc de l'art. Elles s'enrichissent de l'évolutionnisme darwinien, qui propose de montrer comment l'art participe de la nature de l'homme en tant qu'espèce et de 
son évolution : il s'agit d'expliquer les aptitudes esthétiques en tant que compétence biologique parasite ou en tant qu'aptitude élaborée d'adaptation à un milieu, avant d'analyser en quoi les représentations esthétiques illustrent, exemplifient ou modélisent le jeu de forces biologiques. Au nom d'un naturalisme philosophique et d'un positivisme scientifique, on décrète donc que le substrat ultime des faits mentaux est physique, naturel, et que les déterminismes ultimes sont génétiques. Dans le cadre de ce que Quine (1977a, p.105) nomme une "épistémologie naturalisée », le seul ancrage théorique de compréhension de notre savoir, son fondement, doit être celui de la cognition humaine, en tant qu'elle est phénomène naturel explicable in fine par les sciences, sans recours à des modèles externes métaphysiques ou linguistiques, ce qui impose à l'esthétique «d'employer les ressources des sciences de la nature »

Avec le support d'autres champs novateurs, comme l'écopoétique ou la philosophie de l'animal, qui confrontent la philosophie esthétique à des champs aussi éloignés en apparence que l'éthologie et les sciences environnementales, c'est à un décentrement disciplinaire et épistémologique massif que nous assistons. Au nom de ce paradigme, qui relève pour Jean-Marie Schaeffer (2007) de la «fin de l'exception humaine », il incomberait à la philosophie esthétique de proposer la naissance d'une nouvelle science de l'art. Assistonsnous, en réponse à l'artialisation de la nature, à une naturalisation de l'art? En se confrontant frontalement aux études culturelles comme aux paradigmes antérieurs, depuis la psychanalyse jusqu'à l'historicisme, les théoriciens de la naturalisation de l'art se sont opposés aux postures défendant l'insularité et le caractère différentiel des pratiques artistiques ; 
ils ont fourni au contraire un cadre inclusif de compréhension, propre à faire bouger les frontières universitaires et sociologiques de la recherche aussi massivement que son espace disciplinaire. Peut-on alors parler, à la suite du spécialiste américain Jonathan Gottschall (2008b), de «nouvelles humanités » (new humanities), justifiées par l'échec supposé des sciences humaines face aux exigences scientifiques dans le cadre desquelles elles avaient déclaré s'inscrire au moment du linguistic turn?

Cette naturalisation de l'art offre des propositions, épistémologiques autant qu'institutionnelles, séduisantes, dans la mesure où elles permettent de justifier la place de la philosophie esthétique et de la théorie littéraire au sein de l'institution, et, plus généralement, celle du créateur dans la société. Mais elle s'est exposée à de virulentes critiques, accusations de réductionnisme, de conservatisme, d'utilitarisme, d'essentialisme, de scientisme, etc., sur lesquelles je voudrais revenir.

\section{Les présupposés épistémologiques du darwinisme littéraire}

Rappelons tout d'abord que l'évolutionnisme cognitif participe à la fois d'un naturalisme philosophique et d'un positivisme scientifique : le substrat ultime des faits mentaux est physique, naturel, et les déterminismes ultimes sont génétiques. L'ancrage théorique de compréhension de notre savoir, son fondement, doit être celui de la cognition humaine, en tant que phénomène naturel explicable in fine par les sciences, sans 
recours à des modèles externes métaphysiques ou linguistiques.

Dans ce spinozisme total qui renvoie à une forme de matérialisme critique (voir Guerin, p. 146 sqq) et qui, en un sens, équivaut pour notre génération au marxisme de l'aprèsguerre, il n'y a de substrat que strictement biologique aux faits mentaux, et les évolutions cérébrales sont déterminées par des mécanismes propres à l'évolution augmentée de la théorie mendélienne: variation individuelle aléatoire, pression environnementale, sélection génétique et transmission des caractéristiques adaptatives optimales. Ici, la conscience, la religion comme la littérature sont des produits de l'évolution neuronale, gouvernés par la nécessité de maîtriser un environnement humain complexe et dynamique, à la différence des conduites instinctuelles ou de la simple adaptation à un environnement figé et répété prêtée à Homo sapiens : le propre de l'homme en tant qu'espèce, c'est la fluidité cognitive et la plasticité neuronale (voir Mithen, 1996).

Les conséquences d'un tel mouvement de «naturalisation de l'être humain» (Lageira, p. 50) sont considérables, et il importe de les préciser. D'abord, en notant que cette naturalisation de l'esthétique n'est pas assimilable à un retour du « psychologisme » au sens traditionnel ou à la théorie d'une évolution de la littérature au sens de Brunetière, dont on se souvient qu'il avait appliqué le modèle darwinien à la logique des genres littéraires (voir Compagnon, 2001). Comme chez Quine, où la naturalisation de l'épistémologie constitue un cadre d'analyse majeur, nous sommes dans le contexte d'une psychologie envisagée «de manière non psychologique » (Laugier, 2010, p. 24), c'est-à-dire une psychologie anti- 
intentionnaliste, antimentaliste, où il n'y a pas à "interpréter » des expressions privées dans un supposé «langage mental» abstrait qui aurait sa logique propre, mais simplement à classer des faits empiriques selon une démarche scientifique déductive. Si l'on reprend l'opposition formulée par Wilhelm Dilthey entre «sciences de la nature » (Naturwissenschaften), produisant des explications, voire des prédictions, à l'aide de démonstrations expérimentales ou de raisonnements mathématisables, et «sciences de l'esprit» (Geisteswissenschaften), productrices de compréhension, la psychologie évolutionniste de la littérature a vocation à être réintégrée dans les sciences en général et à produire non des interprétations, mais des explications des faits esthétiques. Il s'agit, lorsqu'on parle de la psychologie de l'art, de la fonder sur l'observation empirique, voire sur l'expérimentation, et de se garder de tout discours endogène. Certains théoriciens durs comme Harold Fromm (2009) sont au demeurant proches de ce qu'on appelle le «matérialisme éliminationniste » (eliminative matierialism), représenté notamment par Daniel Dennett ${ }^{1}$ - qui renvoie les faits mentaux à un substrat physiologique hors de portée du sens commun, y compris les qualia (effets subjectifs de nos sensations et expériences), la morale ou la religion - et Richard Dawkins ${ }^{2}-$ l'inventeur de la théorie des mèmes, c'est-à-dire d'une conception des faits artistiques et de la culture en général comme des « unités repliables, supports d'éléments formels ou de contenus stylisés » (Morizot, 2012b, p. 185), soumises aux

\footnotetext{
1 Dennett est professeur de philosophie à la Tufts University, dont il codirige le Center for Cognitive Studies. Sur sa théorie des propriétés mentales, voir Dennet (1991); sur ses vues adaptationnistes: Dennet (1995); sur son athéisme : Dennet (2006).

2 Biologiste et éthologiste, Dawkins est professeur émérite au New College d'Oxford. Voir Dawkins (1976, 1982 et 2006).
} 
lois de la sélection naturelle. Sans aller jusque-là, la pensée "sociobiologique », pour reprendre un concept très utilisé par les «darwiniens littéraires ${ }^{3}$ », tend à réfuter toute interprétation (ou du moins toute interprétation internalisante), toute spéculation sur la valeur particulière et individuelle des œuvres ou sur la personnalité de l'auteur, au nom d'une logique de la très longue durée (le point de départ de l'histoire littéraire cognitiviste, ce n'est pas Homère mais la «révolution humaine », quelque part entre -100000 et -30000 , affirme le fondateur des études littéraires darwiniennes, Joseph Carroll [2011, p. 26]) ou d'une logique quantitative (pour J. Gottschall, « une chose qu'offre la littérature, ce sont des données. Des données rapides, inépuisables, interculturelles et à faible coût » [cité par Max]). On peut songer ici au concept de big data dans le monde numérique : la quantité de données empiriquement disponibles est si importante que son examen quantitatif suppléerait toute théorie. Il s'agit, poursuit Gottschall, de "produire des «hypothèses littéraires qui conduisent à des prédictions vérifiables dans la réalité empirique» (2008b, p.64). Il est alors question de proposer une description empirique, si ce n'est expérimentale, de l'art, ambition qui se propage à d'autres disciplines - et notamment, si l'on en croit Gerald Prince (2006), à la narratologie postclassique - et dont résultent soit des formes de description du fait littéraire tendant à refuser toute spéculation sur les cas particuliers, privés, au nom des faits généraux et massifs, soit, quand on s'intéresse à des faits singuliers, au rejet de toute spéculation esthétique au profit de la simple description d'interactions censées être gouvernées par les nécessités de l'espèce et faisant

3 Expression forgée par le biologiste américain Edward 0. Wilson. Voir D. T. Max (2005). 
sens en série. D'où ce que J. Carroll considère comme une modestie épistémologique : les textes littéraires ne peuvent pas être «décryptés» dans un autre code, car la théorie évolutionniste suppose que les textes sont des outils culturels qui opèrent sur des comportements ordinaires avec un langage ordinaire, qu'ils incorporent une compréhension de la nature humaine au niveau de M. Tout-le-monde (folk understanding [voir Carroll, 2011, p. 146, et Guerin, 2010, p. 146]). Si «la plupart des textes peuvent être correctement compris à un niveau ordinaire de savoir et de langage », ils doivent donc être interprétés à travers le langage ordinaire. Les darwiniens, au contraire des poststructuralistes, s'interdisent par conséquent l'utilisation de «systèmes de signes préfabriqués » dans lesquels ils traduiraient le contenu des textes (voir Carroll, 2011, p. 29). De la même manière que l'on cherche à éviter ce qu'on a pu appeler chez les wittgensteiniens l'illusion de l'intériorité, on va éviter ce que Quine nommait « le mythe de la signification » (1877a ; voir Laugier, 1992, p. 93 sqq.), l'idée que la signification fait partie d'un discours qui dépasse les données dont nous disposons pour parler du langage, en réinscrivant au contraire dans des déterminismes comportementaux naturels les représentations textuelles, qui n'ont ni substance mentale, ni mécanique propre.

Une autre conséquence de cette position épistémologique, c'est qu'il n'y aurait pas plus de logique formelle ou d'historicité propre aux productions symboliques que d'autonomie ontologique, ou même fonctionnelle, de la littérature, celle-ci perdant ainsi toute spécificité. Il n’y a plus de raison de circonscrire un "empire dans un empire », et, pour le dire avec un autre vocabulaire, l'évolutionnisme cognitif s'intègre au paradigme général que J.-M. Schaeffer qualifie de 
«fin de l'exception humaine» (2007, p.185-200). Celui-ci consiste à refuser toute conception anthropocentrée, téléologique ou essentialiste de l'homme (voir p. 201), pour examiner la prétendue "humanité » «à la lumière des contraintes qui la régissent comme espèce biologique ». Notons au passage que, pour Schaeffer comme pour tous les théoriciens de l'évolutionnisme, il n'y a pas d'opposition entre ordre culturel et ordre naturel, puisque la culture est un attribut «naturel» de l'espèce humaine; il s'agit au contraire d'admettre l'existence et de montrer le sens des interactions entre dispositions biologiques et dispositions culturelles - ce qu'on appelle la coévolution génético-culturelle ${ }^{4}-$, ou encore entre ressources et contraintes collectives, d'une part, et aptitudes individuelles particulières, d'autre part (voir Schaeffer, 2007, p. 249). On remarquera ici que cet antiessentialisme est à distinguer à la fois de l'historicisme au sens traditionnel du terme et des philosophies de l'existence: si l'homme n'a pas d'essence, c'est en tant qu'il est un animal, un être biologique et génétiquement modelable, constituant des lignées en constante évolution, et non en tant qu'il transcende par sa liberté son identité biologique (voir Schaeffer, 2007, p. 198-199). Il s'agit donc de chercher les raisons ultimes des productions textuelles symboliques non pas dans les normes culturelles et leurs déterminations, mais dans une "nature humaine » d'ordre génétique qui englobe la culture. On voit ici la puissance de rupture de ces théories : c'est par l'adaptation naturelle que nous devons rendre compte de l'ontologie des

\footnotetext{
4 Démarche qui insiste sur la plasticité adaptative du cerveau, configurée par la pression adaptative, et qui va jusqu'à postuler l'existence d'une sélection neuronale pour expliquer les phénomènes bioculturels. Voir Schaeffer, 2007, p. 341-343.
} 
représentations, c'est par elle que se fait le lien entre la littérature et la réalité. Joseph Carroll (2011, p. 20), s'appuyant sur les travaux de l'éthologue Konrad Lorenz, affirme ainsi que " les sens et l'esprit humain ont accès à la réalité parce qu'ils ont évolué dans une relation adaptative avec un environnement physique et social au sujet duquel l'organisme avait de manière urgente le besoin d'acquérir des informations ».

Prenons quelques exemples précis de ces analyses, en commençant par un cas provocateur : dans un essai intitulé Les Ovaires de Madame Bovary (2005), David et Nanelle Barash mettent en relation les représentations amoureuses de la littérature romanesque avec une série de problèmes d'adaptation : la sélection du meilleur partenaire, l'adultère et ses enjeux en termes génétiques pour l'espèce, l'éducation des enfants et les choix optimaux de la famille. La logique romanesque de Madame Bovary consiste à mettre en scène de la stratégie d'optimisation adaptative du personnage éponyme, une Emma gouvernée par ses ovaires pour trouver un meilleur partenaire que Charles. Donnons un autre exemple: J. Gottschall, dans Le Viol de Troie (2008a), défend l'idée que L'Iliade nous raconte les combats d'êtres humains renvoyés à leur nature animale: "Homère montre que les hommes au combat s'abaissent quasiment au niveau des quadrupèdes et se préoccupent uniquement des processus animaux de conservation et de destruction de la vie » (p. 162) ; ce sont « des singes tueurs surdoués, qui mettent en œuvre leurs magnifiques technologies et leurs instincts de coopération pour traverser les mers et dépouiller d'autres hommes de tout ce qui leur est précieux: leurs richesses, leurs femmes, leurs vies » (p. 163) ; tous agissent inconsciemment en accord avec «la directive première de la vie : croître et multiplier». Mais pour 
Gottschall, Homère montre aussi la manière dont les êtres humains cherchent à dépasser cette animalité, y réussissant parfois (Achille restituant à Priam le corps d'Hector), échouant souvent (voir p. 64) : en ce sens, l'épopée serait non seulement une thématisation, mais une véritable expérience de pensée littéraire quant aux capacités d'évolution de l'homme. Les questions morales souvent placées au cœur du roman n'échappent pas à une telle déconstruction biologisante, comme l'illustre l'important ouvrage de William Flesch, Comeuppance (2008), consacré au "châtiment mérité », aux signaux coûteux, à la punition altruiste et aux autres composantes biologiques de la fiction. Flesch explique lui aussi l'attirance humaine pour la fiction par une version évolutionniste de la théorie des jeux sociaux. Le besoin de fiction vient du fait que l'homme est un animal social. Partant, il s'agit, d'une part, « de traiter les récits comme manifestant un enregistrement exact des formes humaines de coopération » et, d'autre part, de faire de l'œuvre « un objet dont la coopération humaine a besoin et dont elle apprécie la valeur » (Flesch, p. 182). «Les récits racontent des signaux » (au sens de la théorie évolutionniste) " et les récits sont des signaux» (p. 124). Pour Flesch, notre appétence pour le récit s'explique par la nécessité que nous ressentons de surveiller et de nous approprier les formes de réciprocité : en lisant, le lecteur acquiert une aptitude (fitness) à la compréhension et à l'échange dans la relation sociale, une capacité d'empathie, cette dernière étant comprise comme une réciprocité indirecte qui rend chaque individu plus intéressant et plus attractif, tout en le dotant d'une meilleure capacité d'interaction sociale. Être capable de connaître un roman et de s'intéresser à lui manifeste notre capacité à nous impliquer dans des activités altruistes. En découlent des considérations 
sur l'intérêt des romans: "Nous nous intéressons au compte rendu narratif de ce que les gens font à d'autres gens parce que nous nous intéressons au fait de savoir s'ils les traitent de manière égoïste ou altruiste. » (p. 155-156) Altruiste ici diffère de généreux: il est altruiste de punir l'autre pour sa faute, comme il est égoïste de négliger l'obligation de punir les fautifs. En somme, nous nous intéressons dans le roman à ce qui est « prosocial », la vengeance, par exemple, en tant qu'elle est une punition altruiste (Achille, Hamlet, Batman). Alors que la situation de la littérature moderne, celle d'un l'art pour l'art autonomisé et rompant avec les mots et les valeurs de la tribu, pourrait sembler irréductible à une telle fonctionnalité, parce qu'elle place au centre de la scène littéraire un individu marginalisé, un écrivain provocateur et révolté, et le témoignage d'une irréductible singularité, Flesch parvient très habilement à réintégrer l'écrivain moderne, conçu comme celui qui assume la douleur et la difficulté d'être conscient du monde et qui met en partage cette aptitude par un sacrifice altruiste (p. 147) - jusqu'à citer Michel Leiris et sa conception de la littérature comme tauromachie. Flesch explique "l'esthétique » de la modernité en tant que complexification et alourdissement extrême du signal, et comme le lieu où sont mises en scène des formes de coopération raffinée et prosociale. Dans le même esprit et en reprenant cette théorie du "châtiment mérité », Blakey Vermeule (2011) analyse Middlemarch, de George Eliot, en montrant que le génie de la romancière a été de construire son roman non sur les bons sentiments et les logiques propres à la reconnaissance, mais sur l'obscurité morale de l'homme et son désir de punir les autres pour leurs méfaits. Pour généraliser la pensée des évolutionnistes, notre intérêt pour les romans s'explique non par la quête d'une morale individuelle, 
mais plutôt par celle de la morale collective, elle-même surdéterminée par la logique de l'espèce, et non par celle des individus particuliers. Selon un paradoxe déjà analysé par Kant, nos aptitudes antisociales font partie de notre existence sociale. On voit à nouveau les accointances d'une telle théorie avec la vision axiologique de Thomas Pavel dans La Pensée du roman, pour qui le roman joue le rôle d'une boîte à outils pour résoudre un problème axiologique : le roman " pose surtout, et avec une acuité inégalée, la question axiologique qui consiste à savoir si l'idéal moral fait partie de l'ordre du monde [...]. Dans le roman, [...] la question axiologique revient à se demander si pour défendre l'idéal, l'homme doit résister au monde, s'y plonger pour y rétablir l'ordre moral ou enfin s'efforcer de remédier à sa propre fragilité, si, en d'autres termes, l'individu peut habiter le monde où il voit le jour » (p. 46-47).

Une autre piste d'analyse évolutionniste des œuvres littéraires est l'analyse d'ordre quantitatif, illustrant l'empirisme revendiqué des darwiniens littéraires comme leur méthode vérificationniste. J. Gottschall, dans Literature, Science, and a New Humanities (2008b, p. 91 sqq.; voir le bilan de N. Easterlin, 2009), part ainsi de l'hypothèse évolutionniste selon laquelle les protagonistes femelles des récits devraient défendre leur progéniture plutôt que celle des autres; elles devraient choisir des hommes physiquement attirants, dotés d'un statut matériel ou social élevé ; elles devraient être moins actives et moins héroïques d'un point de vue physique que leurs homologues mâles (car dans la majorité des espèces sexuées, les mâles ont des conduites de prise de risque et de recherche de statut dans le groupe qui les exposent à l'alternative très clivée entre reproduction prolifique et mort possible); enfin, les lecteurs devraient préférer des personnages de mâles actifs 
et physiquement héroïques. Gottschall confronte cette hypothèse à un corpus de 658 contes issus de cultures différentes et en tire plusieurs conclusions probantes selon lui : les rôles des héroïnes femelles sont en général excentrés par rapport à ceux des personnages masculins; elles sont en majorité jeunes et belles; elles cherchent à se marier et y arrivent à la fin de l'histoire; elles recherchent d'abord la gentillesse (prosocial) chez le héros, et elles se dévouent ensuite au bien et à la richesse de leur parenté, mais choisissent moins souvent la voie d'une lutte physique active que leurs homologues masculins. Gottschall déduit de ses statistiques des universaux empiriques et les utilise ensuite contre les analyses féministes qui défendent au contraire la dimension culturelle et purement occidentale de nos représentations de la femme : les différences sexuelles examinées en croisant leurs représentations culturelles sont cohérentes et stables, et correspondent à ce que l'on peut attendre des comportements des mammifères sexués (2008b, p 125). Si le critique américain se défend d'être essentialiste ou antiféministe, il réfute les théories culturalistes des genres comme empiriquement inexactes, tout en concédant que les différences statistiquement observées ne nient pas la possibilité d'une action culturelle et d'une plasticité de l'espèce face aux déterminismes biologiques.

\section{Enjeux universitaires et philosophiques de ces disciplines émergentes}

Une telle analyse illustre suffisamment les enjeux des interprétations darwiniennes de la littérature. Dans le contexte américain des études culturelles, elles rompent avec les cadres explicatifs traditionnels, culturalistes, de la critique et leurs 
sous-entendus idéologiques, puisqu'elles réintroduisent au cœur de la réflexion l'idée d'une nature humaine, certes évolutive et non prédéterminée. Dans le contexte français, la rupture se fait par rapport à un autre paradigme critique dominant, celui du formalisme, qui affirme l'autonomie des structures linguistiques. L'enjeu est d'abord épistémologique, car les darwiniens justifient leur démarche par un certain échec des sciences humaines face aux exigences scientifiques dans le cadre desquelles elles déclarent s'inscrire: il s'agirait de dépasser les apories d'une analyse littéraire fondée sur des modèles linguistiques, culturels ou psychanalytiques, et son incapacité rédhibitoire à devenir un outil de prédiction empirique, malgré les jeux structuralistes avec les cases blanches (voir Gefen, 2006) ou les téléologies littéraires marxistes.

L'idée d'une faillite méthodologique des humanités traditionnelles par refus de l'expérimentation conduit J. Gottschall (2008b), on l'a dit, à parler de «nouvelles humanités », d'humanités bénéficiant de l'apport des sciences $\mathrm{du}$ vivant pour proposer des analyses empiriques. Cette proposition a suscité de virulentes réactions, comme celle du neuroscientifique anglais Raymond Tallis (2011a), qui parle de neurotrash au sujet des postures éliminationnistes de Daniel Dennett et Richard Dawkins. Dans son ouvrage Aping Mankind (2011b), Tallis désigne, non sans caricaturer, deux dérives réductionnistes des sciences cognitives: d'une part, la neuromania, c'est-à-dire l'idée que l'activité cérébrale serait la condition suffisante de la conscience humaine et l'unique clef de notre comportement (par exemple, une célèbre étude de détection des régions du cerveau impliquées dans l'amour grâce à l'imagerie par résonance fonctionnelle magnétique ; 
voir Bartels et Zeki) ; d'autre part, la Darwinitis, c'est-à-dire l'idée que la théorie de l'évolution expliquerait non seulement l'origine de l'espèce humaine (ce que Tallis admet en bon darwinien), mais aussi le comportement de l'homme et la nature des institutions sociales.

Tallis est loin d'être le seul à brandir la hache de guerre. Pour résumer les polémiques menées notamment dans les revues Style (voir Eibl et Mellmann) et Poetics Today (vol. 30), les critiques adressées aux évolutionnistes se font au nom d'une position autonomiste défendant l'insularité de l'art et le caractère différentiel des pratiques artistiques, au nom soit de la liberté (en faisant de l'art l'autre de la société et de l'animalité humaine), soit d'un scepticisme épistémologique quant aux inférences produites en référence au passé inaccessible de l'homme préhistorique, soit de réticences face à l'immaturité de la discipline ou à ses ambitions réductionnistes. Ainsi, la critique suisse Marie-Laure Ryan insiste sur le caractère spéculatif des théories de la narration comme adaptation: "puisque nous n'avons pas à notre disposition un spécimen de Néanderthalien ou d'homme de Cro-Magnon, nous ne pouvons comparer la capacité du genre humain à raconter des histoires à divers stades de son évolution culturelle et biologique, et nous en sommes réduits à des spéculations concernant le rôle de la narration dans le développement de l'organisation sociale typique des êtres humains » (p. 481). Il serait, à ce titre, aisé de dénoncer des transferts conceptuels mal maîtrisés et faisant polémique, comme par exemple le rôle hypothétique des neurones miroirs dans les comportements altruistes, étendu depuis les macaques et les bonobos jusqu'aux sociétés humaines évoluées (notamment par Frans de Waal). Un tel manque de finesse conceptuelle rendrait ces doctrines 
naturalisantes inaptes à dire les variations historiques et génériques particulières sans les réduire à des universaux invariables 5 . Or, pour les darwiniens littéraires, cette réduction est inévitable, car consubstantielle à toute réelle production de savoir ; pour eux, comme pour Quine ou Dewey, nous n'avons rien à perdre à embrasser une conception naturaliste et comportementaliste des faits signifiants. La question n'est pas de savoir si les darwiniens réduisent les textes, mais comment. Ils le font, répond J. Carroll, en fournissant un «cadre exhaustif» (comprehensive framework) pour la comparaison entre les perspectives des auteurs, l'organisation du sens dans les textes, et les réponses des lecteurs (voir Carroll, 2011, p 2930, et Mellmann, 2001, p. 310).

Les critiques ne sont pas qu'épistémologiques: pour J. Gottschall, une "critique politique humaniste standard» (2004, p. 214) - autrement dit, une résistance politiquement motivée - est à l'œuvre contre la psychologie évolutionniste ou, si l'on veut, contre son scientisme et son déterminisme. Aux États-Unis, le combat a d'abord été mené sur le front des études culturelles: Michelle Scalise Sugiyama (2006-2007) raconte ainsi que, lorsqu'elle a affirmé dans sa thèse que les personnages littéraires étaient des représentations de la psychologie humaine évoluée et que l'analyse littéraire devrait par conséquent se fonder sur la compréhension de la psychologie évolutionniste, elle fut accusée de réductionnisme et prit plus d'un an pour constituer un jury de thèse à l'université de Californie, Santa Barbara. Elle-même met d'ailleurs en garde contre le risque que la psychologie

5 C'est, par exemple, l'avis de Tony Jackson (2000, p. 329 et 341) et de Frank Kelleter (2007). K. Mellmann (2011, P. 310) s'élève contre cette assertion. Sur la position, mesurée de J.-M. Schaeffer, voir 2009, p. 37-43. 
évolutionniste se contente de proposer un schéma interprétatif universel face aux œuvres, de dévoiler rituellement des mécanismes inconscients et profondément enfouis, à l'instar du freudisme contre lequel elle s'est construite. "Les récits ne se contentent pas de refléter des problèmes d'adaptation et les mécanismes cognitifs apparus au cours de l'évolution pour les résoudre. Les adaptations complexes sont facultatives: elles sont sensibles au changement environnemental et capables de générer des réponses différentes à des facteurs environnementaux différents. Les récits manifestent la nature facultative de notre psychologie évoluée. ${ }^{6}$

On a également reproché aux darwiniens littéraires leur utilitarisme, qui serait celui du darwinisme social, accusation de fatalisme contre laquelle J. Gottschall se défend: «l'étude évolutionniste du comportement et de la psychologie humaine n'est pas [...] une idéologie du pessimisme, du défaitisme ou de la défense conservatrice du statu quo » (2008b, p. 34). Elle obéit $\mathrm{au}$ contraire à l'impératif delphique Connais-toi toi-même, l'apprentissage de la liberté se faisant dans la connaissance du déterminisme biologique: "c'est la vieille bataille des culturalistes contre les biologistes, qui remonte au combat de Boas et de ses élèves contre le darwinisme » (p. 130). Pour les contempteurs de l'évolutionnisme, la naturalisation normative que cette doctrine sous-tend dissimulerait une pensée inégalitaire de droite. L'accusation a été portée, notamment, par Patrick Hogan (2003a) contre l'ouvrage de Randy Thornhill et Craig Palmer, Une histoire naturelle du viol (2000), qui présentait la propension des hommes à commettre des viols

${ }^{6}$ Michelle Scalise Sugiyama s'avoue proche ici des positions de N. Easterlin (2001). 
comme une adaptation évolutive. Gottschall (2004, p. 206), faisant le point sur ce débat, a souligné que de semblables hypothèses, effectivement très contestables, font l'objet d'un débat au sein même de la psychologie évolutionniste, tandis que Peter Singer (2000) défendait l'existence d'un darwinisme de gauche.

Face à ces critiques qui expliquent le caractère encore marginal et presque sectaire des évolutionnistes dans les facultés américaines, des positions critiques intermédiaires ont été suggérées, par exemple en restreignant les ambitions de la méthode. En fin de compte, juge D. T. Max (2005), «le darwinisme littéraire pourrait nous en apprendre moins sur des livres pris séparément que sur l'objet de la littérature ». Au contraire, la littérature moderne serait non réductible aux problématiques d'adaptation et le filtre de la grille évolutionniste serait trop grossier pour comprendre les stratégies littéraires et les conduites dénaturalisées. Pour J.M. Schaeffer (2009), le modèle animal est utile, mais insuffisant, pour décrire les spécificités humaines: dans la théorie des signaux coûteux, les mécanismes de l'art et de la parade amoureuse sont similaires, mais pas les buts et les fonctions. Il faut selon lui distinguer entre homologie structurelle et identité fonctionnelle, sous peine de s'engager dans une démarche réductionniste. En effet, «la relation esthétique n'est pas une fonction : elle se définit comme une dynamique attentionnelle régulée par l'indice d'attractivité de l'activité attentionnelle elle-même » (p. 33).

Une autre position de repli intéressante est représentée par la critique Katja Mellmann (2011, p.301), avec sa proposition du «lecteur modèle anthropologique » (anthropo- 
logical model reader) : la psychologie évolutionniste ne doit pas s'appliquer à des artefacts comme les histoires et les personnages, mais au producteur et au lecteur; elle ne peut concerner que les vivants. Mellmann est ici très opposée à la vision de Carroll et de Gottschall selon laquelle les problèmes d'adaptation seraient représentés et thématisés dans la littérature. Pour celle-ci, il faut travailler sur des sujets vivants concrets et produire une heuristique des motifs d'excitation (stimulus pattern) propre à la lecture : « au lieu de chercher des analogies (redondantes) entre le comportement des personnages de fiction et celui des êtres humains réels, nous ferions mieux de prêter attention aux isomorphismes possibles entre les structures du texte et les schémas innés de déverrouillage des stimuli » (innate releasing schemata, p. 308), car «les artefacts littéraires [...] agissent comme une sorte pseudo-stimulus sur nos dispositions innées » (p. 309), c'est-àdire sur nos récepteurs naturels de sensations. Une telle analyse, plutôt que de nous conduire à des spéculations utilitaristes, nous permettrait par exemple de décrire la fiction comme « une stratégie particulière basée sur nos adaptations que sont le rire et l'humour» (p. 311 ; voir aussi 2007, p. 264352 et 450-451, et 2009).

Quelques mots pour conclure et dire à la fois ma fascination et ma méfiance. On l'a vu, cette théorie émergente, représentée par une génération entière de «darwiniens littéraires » américains, mais aussi par une école française, offre une explication globale du fait littéraire en rupture avec toute la tradition critique française: pour les darwiniens, le fait littéraire n'est pas marginal dans les conduites humaines, mais central à l'espèce; il n'est pas antisocial mais régulateur; la littérature n'a pas d'ontologie propre ni de logique endogène, 
mais elle est surdéterminée biologiquement; les textes ou leur interprétation ne sont pas un espace de liberté, mais un dispositif cognitif, etc. Cette vision de la littérature fait écho à une prise nouvelle de la littérature sur les conduites sociales dont témoignent tant d'essais relevant du développement personnel et du management de soi (comment X ou Y peut vous aider à changer votre $v^{7} \mathrm{e}^{7}$ ), ce qui explique le caractère extrêmement populaire des travaux d'Alan Palmer, de Mark Turner ou de Jonathan Gottschall, qui n'hésitent pas à nous expliquer, pour citer le titre d'un article de Gottschall, " pourquoi la fiction est bonne pour nous ». Assurément, il me semble que les outils proposés sont beaucoup plus fins que ceux des neurosciences: alors que la stylistique ou la narratologie cognitive sont encore embryonnaires et que les travaux d'investigation médicale du cerveau esthétique sont encore balbutiants et inaptes à décrire la complexité et la variété des effets de la littérature, le paradigme évolutionniste produit des catégories descriptives puissantes et ordinaires, formulées dans un langage commun, ainsi qu'une généalogie convaincante de la nécessité de ce processus étrange et pourtant si profondément définitoire de l'homme (Aristote) qu'est la fiction. Les études littéraires cognitives qui émergent rhétorique $^{8}$, poétique ${ }^{9}$ grammaire ${ }^{10}$, stylistique ${ }^{11}$, narrato-

7 Par exemple, Alain de Botton (1997), qui joue, il est vrai, la carte de l'humour, ou Roger Housden (2001), dans une anthologie dont l'auteur a produit d'autres recueils à visée analogue : dix poèmes pour dire adieu, pour vous libérer, pour ouvrir votre cœur. De même Brigitte Méra (2009) livre-telle une très sérieuse leçon de management balzacien.

8 L'expression «rhétorique cognitive » apparaît au milieu des années 1970 dans un article de Dan Sperber (1975). Citons, entre autres, Mark Johnson et George Lakoff (1980), Mark Turner et G. Lakoff (1989) et M. Turner (1997). 
logie ${ }^{12}$, sémiotique ${ }^{13}$ - peuvent se construire largement à partir de cette archéologie du fait artistique autant qu'à partir de résultats observationnels avec des bénéfices évidents pour la littérature: de même que les neurosciences permettent une objectivation des connaissances empiriques et offrent des éclairages fonctionnels sur la cognition (les bases neurophysiologiques de la lecture, de la cognition, etc.), de même le paradigme évolutionniste facilite la réinsertion de la littérature dans les processus cognitifs communs, en nous proposant au passage de faire l'épreuve d'un nouveau vocabulaire et de nouvelles problématiques d'analyse du texte. Un autre intérêt, exemplifié par les travaux de J. Gottschall (2004), mais aussi par ceux, moins immédiatement biologisants, de P. Hogan (2003b, 2006 et 2011), est de relancer la critique à la recherche d'universaux humains par le biais de la littérature, quête à laquelle nous avions largement renoncé. Ainsi, dans l'évolutionnisme affectif de Hogan et sa théorie des archétypes de l'imaginaire, on pourrait dégager des «prototypes émotionnels» (emotion prototypes) fondés sur

\footnotetext{
${ }^{9}$ Le terme de « poétique cognitive » a été forgé au début des années 1990 par Reuven Tsur (1992). Citons également Peter Stockwell (2002), Joanna Gavins et Gerard Steen (2003), Jeroen Vandaele et Geert Brône (2009).

10 Voir Ronald W. Langacker (1987-1991, 1991 et 2008).

11 Voir, pour un premier regard, Elena Semino et Jonathan Culpeper (2003), Michael Toolan et Jean-Jacques Weber (2005) et Michael Burke (2005).

12 Le mouvement a été lancé à la fin des années 1990 par Manfred Jahn (1997). Les points les plus récents sont dus à David Herman (1999, 2002, 2003, 2007 et 2009). Parmi les ouvrages fondateurs: Guy Cook (1994), Monika Fludernik (1996), Catherine Emmott (1997), H. Porter Abbott (2001), Alan Richardson et Francis F. Steen (2002), Alan Palmer (2004), A. Richardson et Ellen Spolsky (2006) et Lisa Zunshine (2006).

13 Voir notamment François Rastier (1991), qui s'appuie sur le fonctionnalisme computationnel de Jerry A. Fodor (1987) et de Zenon Pylyshyn (1984), mais pour qui cette sémantique cognitive doit s'appuyer sur ce qu'il nomme une « herméneutique matérielle » (voir F. Rastier, 2005).
} 
trois rapports différents aux émotions, universaux à valeur transculturelle. Quoi qu'il en soit, la confrontation avec le paradigme darwinien engage un débat vraiment interdisciplinaire. Que l'on adhère ou non à la sociobiologie d'Edward 0. Wilson affirmant l'unité des savoirs dans son livre Consilience : The Unity of Knowledge (1998), on ne peut être que fasciné par l'hypothèse que «la littérature et ses antécédents oraux dérivent d'une disposition, exceptionnellement humaine et propre à l'espèce, à la production et à la consommation de constructions imaginaires verbales. Abolir la barrière méthodologique entre l'expertise humaniste et l'expertise des sciences sociales est susceptible de produire des résultats valant pour les deux domaines. » (Carroll, 2011, p. 35 et 2012) D'où la naissance de nouvelles disciplines comme l'écocritique (ecocriticism) de Harold Fromm ou Glen Love ${ }^{14}$, l'histoire littéraire darwinienne (différente de celle du XIX siècle et de Brunetière) de Tom Dolack (2010), fondée non sur la métaphore des espèces et la réification des formes, mais sur une théorie solide de l'innovation technologique par imitation dans les espèces, ou encore les théories des genres et de la représentation de Robert Storey (1996 ; voir Carroll, 1998).

Avec le postulat interdisciplinaire de l'évolutionnisme littéraire, c'est ici la place institutionnelle et universitaire des humanités qui est interrogée. Que l'on conçoive la littérature comme une base de données anthropologique ou comme un laboratoire de psychologie expérimentale - ou de «philosophie expérimentale », pour reprendre un autre concept à la mode -, on pourrait assister à une relégimation de la critique littéraire

14 Voir, par exemple, Glen Love (2003), Cheryll Glotfelty et Harold Fromm (2009) et H. Fromm (2009). 
et à une réintroduction de celle-ci aux cœurs d'autres savoirs. Telles sont par exemple les vertus des théories narrativistes de A. Turner et du tournant cognitiviste en général : «depuis la publication en 1980 de l'influent ouvrage de George Lakoff et Mark Johnson, Metaphors We Live By, les critiques littéraires ont été encouragés par l'idée d'une poétique cognitive - c'est-àdire d'une théorie systématique de l'esprit dans laquelle la littérature n'est pas seulement périphérique, mais centrale dans la compréhension de la psychologie humaine », écrit Richard Van Oort (2003, p. 238). Autrement dit, la littérature n'a pas seulement à importer passivement un vocabulaire conceptuel exogène et la critique n'a pas seulement à rendre compte de ses métadiscours empiriques avec de nouveaux outils scientifiques, mais elle peut servir, différemment de toutes les autres formes de savoir, à penser des processus mentaux et sociaux concrets en se réinscrivant elle-même dans la très longue durée de l'histoire humaine ${ }^{15}$.

\section{Bibliographie}

Аввотт, H. Porter (dir.). (2001), "On the Origins of Fiction: Interdisciplinary Perspectives », SubStance, vol. 30, no 1. BARASH, David P. et Nanelle R. (2005), Madame Bovary's Ovaries: A Darwinian Look at Literature, New York, Delacorte Press.

15 Je suis en complet accord avec les conclusions de M.-L. Ryan (2010, p. 489). 
BARTELS, Andreas et Semir ZeKI. (2000), «The Neural Basis of Romantic Love », NeuroReport, vol. 11, no 17, p. 3829-3834, <http://www.vislab.ucl.ac.uk/pdf/NeuralBasisOfLove.pdf>. BRUNETIÈRE, Ferdinand. (2000 [1890]), L'Évolution des genres dans l'histoire de la littérature, Paris, Pocket, coll. « Agora ». BuRKE, Michael. (2005), « How Cognition Can Augment Stylistic Analysis », European Journal of English Studies, vol. 9, n² 2, p. 185-196.

CARRolL, Joseph. (1998), compte rendu de Robert F. Storey, Mimesis and the Human Animal, $<$ http://cogweb.ucla.edu/Abstracts/Carroll S98.html .

—. (2011), "An Evolutionary Paradigm for Literary Studies", dans Reading Human Nature: Literary Darwinism in Theory and Practice, Albany (N.Y.), Suny Press, p. 13-54.

-, Jonathan GotTSChall, John A. Johnson et Daniel J. KRUGER. (2012), Graphing Jane Austen: The Evolutionary Basis of Literary Meaning, New York, Palgrave Macmillan.

Collectif. (2009), «Cognitive Themes », Poetics Today, vol. 30, no 3.

Compagnon, Antoine. (2001), "Genre, création, évolution», $<$ http://www.fabula.org/compagnon/genre12.php>.

Cook, Guy. (1994), Discourse and Literature, Oxford, Oxford University Press.

DaWkINS, Richard. (1982), The Extended Phenotype, New York, W. H. Freeman.

—. (1990 [1976]), Le Gène égoïste, trad. de l'anglais par Laura Ovion, Paris, Armand Colin.

—. (2008 [2006]), Pour en finir avec Dieu, trad. de l'anglais par Marie-France Desjeux-Lefort, Paris, Robert Laffont.

De Botton, Alain. (1997), How Proust Can Change Your Life, New York, Pantheon. 
DennetT, Daniel C. (1993 [1991]), La Conscience expliquée, trad. de l'anglais (États-Unis) par Pascal Engel, Paris, Odile Jacob.

—. (2000 [1995]), Darwin est-il dangereux?, trad. de l'anglais (États-Unis) par Pascal Engel, Paris, Odile Jacob.

-. (2006), Breaking the Spell: Religion as a Natural Phenomenon, Londres, Allen Lane.

DoLACK, Tom. (2010), «Imitation and Literary Evolution», Politics and Culture, no 1 ,

$<$ http://www.politicsandculture.org/2010/04/29/imitation -and-literary-evolution/>.

EASTERLIN, Nancy. (2001), « Hans Christian Andersen Fish Out of Water », Philosophy and Literature, vol. 25, n 2, p. 251-277.

-. (2009), " "Literature, Science, and the New Humanities" (Review) ", Philosophy and Literature, vol. 33, no 1, p. 230-233. EIBL, Karl et Katja MELLMANN. (2008), «Misleading Alternatives », Style, vol. 42, nos 2-3, p. 166-171.

Eммотт, Catherine. (1997), Narrative Comprehension: A

Discourse Perspective, Oxford, Oxford University Press.

FLESCH, William. (2008), Comeuppance: Costly Signaling, Altruistic Punishment, and Other Biological Components of Fiction, Cambridge (Mass.), Harvard University Press.

FLUDERNIK, Monika. (1996), Towards a «Natural » Narratology, Londres, Routledge.

FODOR, Jerry A. (1987), Psychosemantics: The Problem of Meaning in the Philosophy of Mind, Cambridge (Mass.), MIT Press.

Fromm, Harold. (2009), The Nature of Being Human: From Environmentalism to Consciousness, Baltimore (Md.), Johns Hopkins University Press.

Gavins, Joanna et Gerard SteEn (dir.). (2003), Cognitive Poetics in Practice, Londres, Routledge. 
GEFEN, Alexandre. (2006), « Du bon usage des tableaux à double entrée: stratégies taxinomiques et ambitions épistémologiques de la théorie littéraire », dans Marc Escola et Sophie Rabau (dir.), La Case blanche : théorie littéraire et textes possibles, actes du colloque d'Oléron (1418 avril 2003), La Lecture littéraire (Reims), no 8.

Glotfelty, Cheryll et Harold Fromm. (dir.). (2009), The Ecocriticism Reader: Landmarks in Literary Ecology, 2e éd., Athens (Ga.), The University of Georgia Press.

GopNIK, Adam. (2012), "Can Science Explain Why We Tell Stories? ", The New Yorker,

$<$ http://www.newyorker.com/online/blogs/books/2012/0 5/can-science-explain-why-we-tell-stories.html>.

GoTTSCHALL, Jonathan. (2004), "Literary Universals and the Sciences of the Mind», Philosophy and Literature, vol. 28, p. 202-217.

- (2008a), The Rape of Troy: Evolution, Violence, and the World of Homer, Cambridge et New York, Cambridge University Press.

-. (2008b), Literature, Science, and a New Humanities, New York, Palgrave Macmillan.

GUERIN, Wilfred L. et alii. (2010), A Handbook of Critical Approaches to Literature, 6e éd., Oxford et New York, Oxford University Press.

HERMAN, David (dir.). (1999), Narratologies: New Perspectives on Narrative Analysis, Columbus (Ohio), Ohio State University Press.

-. (2002), Story Logic: Problems and Possibilities of Narrative, Lincoln (Nebr.), University of Nebraska Press.

- . (2003), "Stories as a Tool for Thinking », dans id. (dir.), Narrative Theory and the Cognitive Sciences, Stanford (Calif.), CSLI, p. 163-192. 
-. (2007), "Storytelling and the Sciences of the Mind: Cognitive Narratology, Discursive Psychology, and Narratives in Face-to-Face Interaction », Narrative, vol. 15, p. 306-334.

-. (2009), Basic Elements of Narrative, Oxford, Wiley-Blackwell. HoGAN, Patrick Colm. (2003a), Cognitive Science, Literature, and the Arts: A Guide for Humanists, New York et Londres, Routledge.

- (2003b), The Mind and Its Stories: Narrative Universals and Human Emotion, New York, Cambridge University Press.

- . (2006), « A Debate for Making Clear the Worth of Universals: Ninety-Five Theses »,

$<$ http://www.redes.lmu.de/igel/resources/discussions/Hog an theses.pdf $>$.

-. (2011), Affective Narratology: The Emotional Structure of Stories, Lincoln (Nebr.), University of Nebraska Press.

Housden, Roger. (2001), Ten Poems to Change Your Life, New York, Harmony.

JACKSON, Tony. (2000), «Questioning Interdisciplinarity:

Cognitive Science, Evolutionary Psychology, and Literary Criticism », Poetics Today, vol. 21, n 2, p. 319-347.

JaHN, Manfred. (1997), «Frames, Preferences, and the Reading of Third-Person Narratives: Toward a Cognitive Narratology », Poetics Today, vol. 18, no 4, p. 441-468. JoHnSON, Mark et George LAKOFF. (2003 [1980]), Metaphors We Live By, Chicago, University of Chicago Press.

KELLETER, Frank. (2007), "A Tale of Two Natures: Worried Reflections on the Study of Literature and Culture in an Age of Neuroscience and Neo-Darwinism ", Journal of Literary Theory, vol. 1, no 1, p. 153-189, 
$<$ http://www.jltonline.de/index.php/articles/article/view/ 65/258>.

LAGEIRA, Jacinto. (2012), "Artialisation », dans Roger Pouivet et Jacques Morizot (dir.), Dictionnaire de philosophie esthétique, $2^{\mathrm{e}}$ éd. rev. et corr., Paris, Armand Colin, p. 49-50.

LANGACKER, Ronald W. (1987-1991), Foundations of Cognitive Grammar, Stanford (Calif.), Stanford University Press, 2 vol.

-. (1991), Concept, Image, and Symbol: The Cognitive Basis of Grammar, Berlin et New York, Mouton de Gruyter.

-. (2008), Cognitive Grammar: A Basic Introduction, New York, Oxford University Press.

LAUGIER, Sandra. (1992), L'Apprentissage de l'obvie: l'anthropologie logique de Quine, Paris, J.Vrin, coll. «Bibliothèque d'histoire de la philosophie ».

—. (2010), Wittgenstein, le mythe de l'inexpressivité, Paris, J. Vrin, coll. « Problèmes et controverses ».

Love, Glen. (2003), Practical Ecocriticism: Literature, Biology, and the Environment, Charlottesville (Va.), University of Virginia Press.

MaX, D. T. (2005), "The Literary Darwinists », The New York Times Magazine, 6 novembre,

$<$ http://www.nytimes.com/2005/11/06/magazine/06darw in.html?pagewanted $=$ all $>$.

MellmanN, Katja. (2007), Emotionalisierung. Von der Nebenstundenpoesie zum Buch als Freund, Paderborn, Mentis.

-. (2009), "Das "Spielgesicht" als poetisches Verfahren. Elemente einer verhaltensbasierten Fiktionalitätstheorie », dans Thomas Anz et Heinrich Kaulen (dir.), Literatur als Spiel. Evolutionsbiologische, ästhetische und pädagogische Aspekte, Berlin, de Gruyter, p. 65-86. 
- (2011), « Evolutionary Psychology as a Heuristic in Literary Studies ", dans Nicholas Saul et Simon J. James (dir.), The Evolution of Literature: Legacies of Darwin in European Cultures, Amsterdam, Rodopi, p. 299-317.

MÉRA, Brigitte. (2009), La Méthode Rastignac: "La Comédie humaine ", une culture d'entreprise, Paris, Tallandier.

Mithen, Steve J. (1996), The Prehistory of the Mind: A Search for the Origins of Art, Religion, and Science, Londres, Thames and Hudson.

Morizot, Jacques. (2012a), «Cognitif», dans Roger Pouivet et Jacques Morizot (dir.), Dictionnaire de philosophie esthétique, 2e éd. rev. et corr., Paris, Armand Colin, p. 86-89.

- . (2012b), «Évolution et esthétique », ibid., p. 184-186.

PALmer, Alan. (2004), Fictional Minds, Lincoln (Nebr.), University of Nebraska Press.

PARRY, Mark. (2011) «Raymond Tallis Takes Out "Neurotrash" », The Chronicle Review, <http://chronicle.com/article/Raymond-Tallis-Takes-Outthe $/ 129279 />$.

Pavel, Thomas. (2003), La Pensée du roman, Paris, Gallimard, coll. « NRF-essais ».

PRINCE, Gerald. (2006), « Narratologie classique et narratologie post-classique »,

<http://www.vox-poetica.org/t/articles/prince.html>.

PYLYSHYN, Zenon. (1984), Computation and Cognition: Toward a Foundation for Cognitive Science, Cambridge (Mass.), MIT Press. Quine, W.V. O. (1977a [1968]), "Relativité de l'ontologie», dans Relativité de l'ontologie et autres essais, trad. de l'anglais (États-Unis) par Jean Largeault, introduction par Sandra Laugier, Paris, Aubier, coll. « Philosophie», p. 39-81. 
—. (1977b [1968]), « L'épistémologie naturalisée », ibid., p. 83105.

RASTIER, François. (2001 [1991]), Sémantique et recherches cognitives, Paris, PUF, $2^{\mathrm{e}}$ éd. augm.

- (2005), "Sémiotique du cognitivisme et sémantique cognitive : questions d'histoire et d'épistémologie », Texto, $<$ http://www.revue-

texto.net/inedits/rastier/rastier semantiquecognitive.html>.

RiCHARDSON, Alan et Ellen SPOLSKY (dir.). (2006), The Work of Fiction: Cognition, Culture, and Complexity, Aldershot, Ashgate.

RICHARDSON, Alan et Francis F. STEEN (dir.). (2002), « Literature and the Cognitive Revolution », Poetics Today, vol. 23, n 1.

RYAN, Marie-Laure. (2010), «Narratology and Cognitive Science: A Problematic Relation », Style, vol. 44, no 4, p. 469495.

SCALISE SugIYAMA, Michelle. (2006-2007), «The Nature of Literature », Entelechy, no 8, $<$ http://www.entelechyjournal.com/michellescalisesugiyam a.html>.

SCHAEFFER, Jean-Marie. (2007), La Fin de l'exception humaine, Paris, Gallimard, coll. « NRF-essais ».

-. (2009), Théorie des signaux coûteux, esthétique et art, présentation de Suzanne Foisy, Rimouski (Québec), Tangence éditeur, coll. « Confluences ».

SEMINo, Elena et Jonathan CulPEPER (dir.). (2003), Cognitive Stylistics: Language and Cognition in Text Analysis, Amsterdam et Philadelphie, John Benjamins.

SingER, Peter. (2000), A Darwinian Left: Politics, Evolution, and Cooperation, New Haven (Conn.), Yale University Press. 
SPERBER, Dan. (1975), "Rudiments de rhétorique cognitive», Poétique, no 23, p. 389-415.

Stockwell, Peter. (2002), Cognitive Poetics: An Introduction, Londres, Routledge.

STOREY, Robert F. (1996), Mimesis and the Human Animal: On the

Biogenetic Foundations of Literary Representation, Evanston (Ill.), Northwestern University Press.

TALLIS, Raymond. (2011a), « Neurotrash. Humans Are Special», Prospect,

$<$ http://www.prospectmagazine.co.uk/magazine/neurotras h-brain-chemistry-biologism-neurones-darwin $/>$.

-. (2011b), Aping Mankind: Neuromania, Darwinitis and the Misrepresentation of Humanity, Durham, Acumen.

Thornhill, Randy et Craig T. PALmer. (2000), A Natural History of Rape: Biological Bases of Sexual Coercion, Cambridge (Mass.), MIT Press.

Toolan, Michael et Jean-Jacques Weber (dir.). (2005), "The

Cognitive Turn: Papers in Cognitive Literary Studies», European Journal of English Studies, vol. 9, no 2.

Tsur, Reuven. (2008 [1992]), Toward a Theory of Cognitive Poetics, 2e éd., Brighton et Portland, Sussex Academic Press. TuRnER, Mark. (1997), The Literary Mind: The Origins of Thought and Language, New York, Oxford University Press.

- et George Lakoff. (1989), More Than Cool Reason: A Field Guide to Poetic Metaphor, Chicago, University of Chicago Press.

VAN OORT, Richard. (2003), « Cognitive Science and the Problem of Representation », Poetics Today, vol. 24, no 2, p. 237-295.

VANDAELE, Jeroen et Geert BRôNE (dir.). (2009), Cognitive Poetics: Goals, Gains and Gaps, Berlin, Mouton-de Gruyter. 
Vermeule, Blakey. (2011), «A Comeuppance Theory of Narrative and Emotions », Poetics Today, vol. 32, n 2, p. 235253.

WILSON, Edward O. (1998), Consilience: The Unity of Knowledge, New York, Alfred A. Knopf.

Zunshine, Lisa. (2006), Why We Read Fiction: Theory of Mind and the Novel, Columbus (Ohio), Ohio State University Press.

\section{Résumé}

Proposant à la suite de Gottschalll de parler de "nouvelles humanités », les darwiniens justifient leur démarche par un échec des sciences humaines face aux exigences scientifiques dans lesquelles elles avaient déclaré s'inscrire au moment du linguistic turn. En se confrontant frontalement aux études culturelles, comme aux paradigmes antérieurs, de la psychanalyse à l'historicisme, les théoriciens évolutionnistes se sont opposés aux postures défendant l'insularité de l'art et le caractère différentiel des pratiques artistiques en fournissant au contraire un cadre inclusif pour la comparaison entre les perspectives des auteurs, l'organisation du sens dans les textes et les réponses des lecteurs, comme une défense de la place des études littéraires dans le savoir - et donc dans l'institution universitaire. Une telle position s'est exposée à de virulentes critiques (réductionnisme, conservatisme, utilitarisme, essentialisme, scientisme, etc.) sur lesquelles le présent article revient tant elles semblent faire obstacles à un examen serein des propositions disciplinaires avancées, dans toute leur richesse et leur ampleur épistémologique. 


\begin{abstract}
Darwinian critics propose, after Gottschalll, to talk about "new humanities". They justify themselves by putting forth the humanities' failure with regard to scientific demands within which they had claimed their obedience at the time of the linguistic turn. Facing cultural studies head-on, like anterior paradigms, from psychoanalysis to historicism, evolutionary theorists have opposed themselves against all the positions defending art's insularity as well as the differential character of artistic practices by providing, on the contrary, an inclusive framework for comparing the authors' perspectives, the organization of textual meaning, and readers' responses as a defense of the location of literary studies within knowledge and therefore within the academic institution. Such a position has raised violent criticism (reductionism, conservatism, utilitarism, essentialism, scientism and so on) upon which I would like to elaborate, inasmuch as they seem to render impossible a dispassionate examination of the disciplinary proposals put forth, in all their richness and epistemological breadth.
\end{abstract}

